\title{
Unique journal: Bio-Design and Manufacturing
}

\author{
Huayong Yang ${ }^{1} \cdot$ Zhanfeng Cui $^{2}$
}

Published online: 26 February 2018

(c) Zhejiang University Press 2018

When we were asked to consider to launch this new journal, the first question was why? There are so many journals now, why we need to launch another one? When we said 'yes', we have decided to make this journal unique. This is not just another one, this is 'Bio-Design and Manufacturing (BDM)'.

Firstly we promote engineering excellence. Design and manufacturing take the centre space of the scope of the journal. The journal will primarily serve engineers and provide a platform for engineering practitioners and technologists in all engineering branches, civil, chemical, computing, electrical, information, instrumentation, material, mechanical, medical, process and so on.

Secondly we target designing and solving the problems in the new bio-dimension, where no establish methodologies have been established. Even fundamental parameters in the biosystems need to be estimated and changes with time, time constants can be vastly different from conventional process, and new set of restrictions, often not well defined, will have to impose. All these provide interesting and important challenges and need collective research effort.
Thirdly interfacing with life science and medicine is an essential and integrated process in bio-design and manufacture. The exponential advances in life science and biotechnology enhance our understanding on the detailed mechanisms of 'how things work in biosystems', but engineering and bio-manufacture are essential to 'how to make the biosystems work in a controlled way' and to benefit human society.

We welcome contributions from researchers all over the world and hope readers from both academia and industry find the articles published in BDM are interesting, inspiring and useful. We would like to use this journey as a platform to gather the community together and to address important issues related to bio-design and manufacturing. Together we make the journal-BDM-unique and successful.

Huayong Yang; Zhanfeng Cui, Editors-in-Chief, Bio-Design and Manufacturing

Huayong Yang

yhy@zju.edu.cn

Zhanfeng Cui

zhanfeng.cui@eng.ox.ac.uk

1 Chinese Academy of Engineering, Zhejiang University, Hangzhou, China

2 Royal Academy of Engineering, University of Oxford, Oxford, UK 